\title{
Energy Optimization Using a Pump Scheduling Tool in Water Distribution Systems
}

\author{
Karwan A. Muhammed ${ }^{1,2}$, Raziyeh Farmani ${ }^{3}$ \\ ${ }^{1}$ Department of Water Resource, College of Engineering, \\ University of Sulaimani, Sulaymaniyah, Kurdistan Region - F.R. Iraq \\ ${ }^{2}$ Department of Civil Engineering, College of Engineering, Komar \\ University of Science and Technology, Sulaymaniyah, Kurdistan Region - F.R. Iraq \\ ${ }^{3}$ Centre for Water Systems, College of Engineering, Mathematics and Physical Sciences, \\ University of Exeter, Exeter, EX4 4QF, U.K
}

\begin{abstract}
Water distribution management system is a costly practice and with the growth of population, the needs for creating more cost-effective solutions are vital. This paper presents a tool for optimization of pump operation in water systems. The pump scheduling tool (PST) is a fully dynamic tool that can handle four different types of fixed speed pump schedule representations (on and off, time control, time-length control, and simple control [water levels in tanks]). The PST has been developed using Visual Basic programming language and has a linkage between the EPANET hydraulic solver with the GANetXL optimization algorithm. It has a user-friendly interface which allows the simulation of water systems based on (1) a hydraulic model (EPANET) input file, (2) an interactive interface which can be modified by the user, and (3) a pump operation schedule generated by the optimization algorithm. It also has the interface of dynamic results which automatically visualizes generated solutions. The capabilities of the PST have been demonstrated by application to two real case studies, Anytown water distribution system (WDS) and Richmond WDS as a real one in the United Kingdom. The results show that PST is able to generate high-quality practical solutions.
\end{abstract}

Index Terms - Optimization, Pump scheduling, Water distribution systems.

\section{INTRODUCTION}

Large amounts of energy are consumed in the water sector to satisfy increasing demands and regulatory requirements. The main consumption of energy is associated with pumping large amounts of water from sources to distribution systems (MalaJetmarova, Sultanova and Savic, 2018; León-Celi, et al., 2016; Ghaddar, Naoum-Sawaya and Kishimoto, 2015; and

ARO-The Scientific Journal of Koya University Volume VIII, No.1(2020), Article ID: ARO.10635, 12 pages DOI: $10.14500 /$ ARO.10635

Received: 14 February 2020; Accepted: 30 June 2020

Regular research paper: Published: 30 June 2020

Corresponding author's email: karwan.muhammed@univsul.edu.iq

Copyright (C) 2020 Karwan A. Muhammed, Raziyeh Farmani. This is an open-access article distributed under the Creative Commons Attribution License.
Makaremi, Haghighi and Ghafouri, 2017). The environmental footprint associated with these large energy demands also remains a challenge. Therefore, an efficient pump operation to save energy and reduce environmental impact (e.g., carbon emissions) in water supply systems has become a common concern for water utilities. Optimum pump scheduling has been found to be an efficient method to optimize pump energy consumption (Kougias and Theodossiou, 2013 and Giacomello, Kapelan and Nicolini, 2013), which can lead to significant saving in energy without any infrastructure changes to the components of water supply system. Pump scheduling problems are large-scale, non-linear, and highly complex computational optimization problems (Ghaddar, Naoum-Sawaya and Kishimoto, 2015; Choi and Kim, 2019; Amirabdollahian and Mokhtari, 2015; Skworcow, et al., 2014; Tsai, et al., 2008; Behandish and Wu, 2014; and Abdallah and Kapelan, 2017), due to the large number of decision variables and constraints, and several conflicting objectives that need to be considered (Blinco, et al., 2016). The size of solution space exponentially grows with the number of decision variables (Nowak, et al., 2018) and proportionally changes with the number of pumps and the length of simulation period. The number of possible solutions for a problem with $P$ pumps during a $24 \mathrm{~h}$ simulation period snapshot is equal to $2^{\left(P^{* 24}\right)}$. The most widely considered objective function in different approaches is to minimize total operational costs (Kougias and Theodossiou, 2013; Blinco, et al., 2016; Farmani, et al., 2007; Jung, et al., 2014; Menke, et al., 2016; Vieira, et al., 2019; Kurian, et al., 2018; and Odan, Ribeiro Reis and Kapelan, 2015), whereas in some approaches, the problem is formulated as a two-objective optimization problem (Wang, Chang and Chen, 2019; Fayzul, Pasha and Lansey, 2014; and Housh and Salomons, 2018), considering the total number of pump switches as a second objective.

Another important aspect of pump scheduling problems is the representation of a candidate schedule. A specific representation effectively defines how an optimization algorithm handles the problem (López-Ibáñez, Prasad and Paechter, 2011), and how a set of decision variables 
is selected for a pump scheduling problem. Usually, the representation of pump control decision variables can be introduced in two different ways: (1) The implicit way which uses the hydraulic conditions of system components to define the operation of pumps such as simple control rules based on tank water trigger levels (Behandish and $\mathrm{Wu}, 2014$ and van Zyl, Savić and Walters, 2004) or simple control rules with time base (Marchi, Simpson and Lambert, 2016), pump speeds (Hashemi, Tabesh and Ataeekia, 2014 and Zhang and Zhuan, 2018), and pump flow (Bene and Hos, 2012) and (2) the explicit way in which the status of pumps is specified explicitly based on time. The three most common types of explicit pump scheduling are as follows: (1) Duration of pump operation (López-Ibáñez, Prasad and Paechter, 2008); (2) on/off pump status during pre-defined equal time intervals (Costa, de Athayde Prata and Ramos, 2015; NaoumSawaya, et al., 2015, Siew, Tanyimboh and Seyoum, 2016; and Mala-Jetmarova, Barton and Bagirov, 2015); and (3) start/end run times of the pumps (Bagirov, et al., 2013). Alternative representations of pump scheduling may enhance optimization algorithms and are likely to have an impact on the quality of solutions (López-Ibáñez, 2009). A number of decision support tools have recently been developed, each with a particular schedule type, for optimizing the pump operation of water distribution systems (WDSs) including the Pollution Emission Pump Station Optimization tool (Sadatiyan and Miller, 2019); water distribution cost-emission nexus (Stokes, Simpson and Maier, 2014); Darwin Scheduler (Alighalehbabakhani, et al., 2015); and Markov decision processes (Fracasso, Barnes and Costa, 2013).

In all above-mentioned approaches, the tools and models have been developed for specific application purposes and posed as either a single or multiobjective (MO) optimization problem. This paper presents a new powerful model-driven decision support tool called the pump scheduling tool (PST). The PST is a dynamic tool that can handle both implicit and explicit fixed speed pump scheduling types, considering four different pump scheduling formulation options (on and off, time control, time-length control, and simple control [water levels in tanks]) to generate optimum pump operation schedules for water systems. The tool has a dynamic user friendly interface used for simulation of water systems based on: (1) A hydraulic model input file, (2) an interactive interface which can be modified by the user, and (3) a pump scheduling generated by an optimization algorithm. This allows testing different operation options and identifying schedules that are most cost effective. The PST has been developed as an Excel add-in with a user-friendly interface and is an extension of the linkage between the EPANET hydraulic solver and the GANetXL (Savić, Bicik and Morley, 2013) optimization algorithm. The user-friendly interface allows easy parameter setting for a desired pump scheduling type. The GANetXL optimization tool provides the optimization engine for the PST. The problem has been posed as the minimization of energy cost while satisfying a set of hydraulic constraints. The optimization problem can be set as a $\mathrm{MO}$ optimization or single objective optimization to obtain an optimum pump schedule. The structure, feature, and methodology used for the PST development are presented below followed by the application of the tool to two case studies and finally the key findings and future research direction in the conclusions.

\section{DeVELOPMENT OF PST}

To develop an efficient decision support tool (DST) in a generic framework which is applicable and flexible for a range of WDS operation problems, a number of crucial prior considerations should be taken into account. The important prior considerations involved in the PST development are pump scheduling problem requirements, hydraulic simulation model, and optimization method.

\section{A. Pump Scheduling Problem}

Pump scheduling can be defined as the method of choosing which of the available pumps in a WDS are to be utilized and operated during specific times of the day. The main objective is to find the best pump schedule that minimizes total operation costs while satisfying the system performance requirements such as supplying water to the customers at desired pressure, allowing for emergency water level in tanks and full recovery of tank levels by the end of the simulation period.

Pump operation costs include the cost of energy consumed by pumps and pump maintenance costs resulting from the workload forced on pumps. The energy consumption charge is considered as the main objective in most optimization models of pump operation (Mala-Jetmarova, Sultanova and Savic, 2017). On the other hand, maintenance costs, including the costs of repairing and replacing damaged pumps, are measured using a surrogate objective (MalaJetmarova, Sultanova and Savic, 2017), such as the number of pump switches. A pump switch is defined as changing the status of a pump in a time step from off to on or vice versa. Frequent pump switching causes wear and tear on pumps, which subsequently increases maintenance costs (LópezIbáñez, Prasad and Paechter, 2008).

\section{B. Simulation Model and Optimization Algorithm}

It is important to select an appropriate hydraulic simulation model for a real water distribution application (Stokes, Simpson and Maier, 2015). Due to the availability of EPANET simulation models for most real WDS (De Corte and Sörensen, 2013), and the ease of integrating EPANET with optimization algorithms, EPANET 2.0 (Marchi, Simpson and Lambert, 2016 and Rossman, 1999) is considered as a hydraulic solver in the PST development. Advantages of using such a simulation approach are its ability to implicitly handling the equations of conservation of mass and energy and hydraulic constraints that define the flow phenomena as well as having a graphical user interface to visualize the results of simulations. An extended period simulation (EPS) model is conducted to hydraulically simulate the pumping operation of WDS and evaluate the effects of changing water usage overtime. 
Due to the large number of possible solutions in a WDS operation problem as well as the non-linearity of the system, changes made at one component of the system may influence the performance at another. This requires an optimization method that can handle such problems. Heuristic optimization methods, especially genetic algorithms (GAs), are particularly suited for use (Matott, Tolson and Asadzadeh, 2012) and have proved to be effective in the management of WDS problems (Tsoukalas, et al., 2016) specifically those with highly nonlinear combinatorial search spaces, multiple local optima, and many constraints (e.g., pump scheduling problem) (van Zyl, Savić and Walters, 2004 and Walski, Chase and Savic, 2003). GANetXL has been used as the optimization tool in this work.

\section{Problem Formulation IN PST}

The problem is posed as optimum pump scheduling which aims to minimize total energy cost of pumping in water systems. The PST can handle four different pump scheduling representations in a single framework to generate optimum pump operation schedules. The tool has a dynamic userfriendly interface which makes it easy to be used for different types of pump scheduling problems. In addition to automatic optimization task, the integrated model has specific submodels to perform only hydraulic simulation of current schedule of a given problem or manual modification of existing schedule. The formulations of decision variables, objective functions, and constraints in the tool are explained in the following subsections.

\section{A. Decision Variables}

The PST handles the pump configuration decision variables using the two most popular pump status representations: Explicit representation based on time control, timelength control, and on/off at each time step and implicit representation based on simple control (water levels in tanks). The formulations of these four schedule types used in the PST are detailed in the following sections.

\section{Time control}

For time control, the number of pumps operating in each time step is defined as a decision variable and formulated as follows:

$$
T C S=\left[\begin{array}{ccccccc}
t_{j} & t_{(j+1)} & t_{(j+2)} & \cdot & \cdot & t_{(j+M-1)} \\
N A P & N A P & N A P & \cdot & \cdot & \cdot & N A P
\end{array}\right]_{, 0 \leq N A P \leq N}
$$

where, TCS is the time control schedule matrix, NAP is the possible number of combinations of active pumps in each time step, $\mathrm{N}, \mathrm{M}$, and $\mathrm{t}$ are the number of pumps, simulation period, and time step value, respectively.

\section{Time-length control}

A pump schedule can also be explicitly defined by specifying the time during which each pump is on or off. From an operation perspective, explicit pump scheduling based on time-length is considered as a more practical schedule than other schedule representation approaches (Bagirov, et al., 2013). In this schedule representation, the length of time $(T L)$ in which pumps are on and the time when a pump switches on (ST) are considered as decision variables. To simplify the formulation and reduce the solution search space, usually, the maximum number of pump actions is specified beforehand (López-Ibáñez, Prasad and Paechter, 2008). The maximum number of actions allowed per pump during a scheduling period is assumed to be two, since frequent pump switching also causes wear and tear on pumps which subsequently increases maintenance costs [(LópezIbáñez, Prasad and Paechter, 2008). Time-length control schedule matrix is used to define the decision variables.

$$
T L C S=\left[\begin{array}{c}
S_{p_{i}}\left(\operatorname{OPr}_{i}, \operatorname{OPr} 2_{i}\right) \\
\vdots \\
S_{p_{(i+1)}}\left(\operatorname{OPr}_{(i+1)}, \operatorname{OPr} 2_{(i+1)}\right) \\
\vdots \\
\vdots \\
S_{p_{(i+N-1)}}\left(\operatorname{OPr}_{(i+N-1)}, \operatorname{OPr2} 2_{(i+N-1)}\right)
\end{array}\right]
$$

where, $S_{p i}$ is the schedule of $i_{t h}$ pump, and $\operatorname{OPr} 1_{i}$ and $\operatorname{OPr} 2_{i}$ are the first and second operation periods of $i_{\text {th }}$ pump during a $24 \mathrm{~h}$ simulation period. Since the number of pumping actions is two, there are only two operation periods. $O P r$ is an array for storing decision variables, including time when a pump switches on (ST) and the length of time it stays on (TL) (i.e., $\operatorname{OPr} 1=[S T 1, T L 1])$.

\section{On and off}

The configuration of the pump is defined by binary coded chromosomes. The binary notation is used to represent the on/off status of a particular pump in a specific time interval (1: pump is on and 0: pump is off). A decision variable matrix is introduced to store the pump status binary values during the simulation period.

$$
D V M=\left[\begin{array}{cccccc} 
& t_{j} & t_{(j+1)} & \cdot & \cdot & t_{(j+M-1)} \\
S^{P_{i}} & 0 / 1 & 0 / 1 & . & . & 0 / 1 \\
S^{P_{(i-1)}} & 0 / 1 & 0 / 1 & . & . & 0 / 1 \\
\cdot & \cdot & \cdot & \cdot & . & \cdot \\
\cdot & \cdot & \cdot & \cdot & . & . \\
S^{P_{(i+N-1)}} & 0 / 1 & 0 / 1 & . & . & 0 / 1
\end{array}\right]
$$

where, $S^{P_{i}}$ is the schedule of $i_{\text {th }}$ pump, $\mathrm{i}$ is the pump index $(\mathrm{i}=1,2,3 \ldots \ldots N),(N$ is the total number of pumps involved in the optimization), $t_{j}$ is the $j_{t h}$ time step, and $j$ is the time index $(\mathrm{j}=1,2,3 \ldots . M)$.

\section{Simple control}

In this case, the pump operation variables are defined in terms of tank level controls. Each pumping action is linked with a pair of pre-determined water levels (lower and upper trigger levels, as shown in Fig. 1) such that when water falls or reaches the predefined levels, the pumps are switched on 


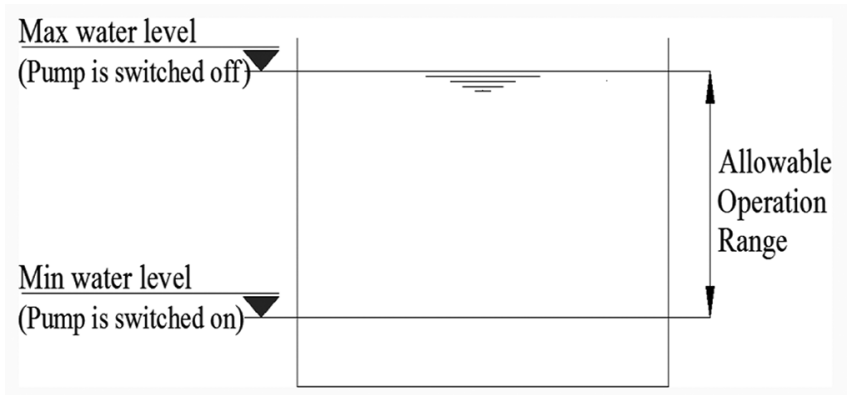

Fig. 1. Trigger levels for a tank.

or off, respectively. This can be done using a subset of simple control rules for trigger levels of the tank and the maximum and minimum water level values are determined by the water level of the full tank and emergency storage requirements. The water levels in the tanks at the start of operation are considered as decision variables that vary between maximum and minimum water levels in each tank.

\section{B. Objective Function}

The optimization model integrated in the PST is posed as a cost optimization model for the pump scheduling problem which aims to minimize the total cost of supplying water, while maintaining a set of system boundaries and constraints. The total energy cost and the number of pump switches (as a surrogate measure for maintenance costs) are considered as two main objectives in the optimization procedure. Such formulation represents a better way of assessing the tradeoffs between the energy costs and the maintenance costs caused by an excessive number of pump switches (Walski, Chase and Savic, 2003). The mathematical formulation of the objective functions is given in Equations 1 and 2.

$$
\begin{gathered}
\operatorname{Minimise}(T E)=\sum_{i=1}^{N} \sum_{t=1}^{M} S_{i, t} \times E_{i, t} \times P_{i, t} \\
\text { Minimise(TNPS) }=\sum_{i=1}^{N}\left(\sum_{t=1}^{M} P s_{i, t}\right)
\end{gathered}
$$

where, TE is the total energy cost of the pumps during the operation period $\mathrm{M}$ (e.g., $24 \mathrm{~h}$ ), $E$ and $P$ are the consumed energy $(\mathrm{kWh})$ and the unit energy cost of the pump operation in time-step $t, \mathrm{~S}$ is the status of the pump $i$, and $N$ is the total number of pumps in the system. TNPS is the total number of pumps switches. PS is the pump switch indicator value for the configuration of each pump between 2 time steps, which is 1 if the pump is active in the current time step (t) and was not active in the previous time step $(\mathrm{t}-1)$, or vice versa.

The tool can be used to solve the problem either as a two objective optimization, considering proposed objective functions in Equations 1 and 2 or as a single objective optimization, in which case a decision can be made whether to consider the number of pump switches as a constraint added to the penalty function or not.

\section{Constraints}

To generate a feasible schedule, a number of system constraints have to be satisfied. In general, the constraints can be divided into hydraulic constraints (system constraints) and operational performance constraints. In the simulation model, the hydraulic simulator, EPANET, implicitly handles hydraulic constraints. For those cases which the hydraulic equations that model flow and energy balance in a network cannot solve (e.g., due to ill conditioned equation systems), a specific error or a warning message (e.g., pumps cannot deliver enough flow or head, and system has negative pressures) is generated by EPANET. The number of those messages is considered as a hydraulic constraint to eliminate those solutions which contain such errors and satisfy a successful hydraulic run.

The two important operational constraints are, water should be supplied to customers with adequate pressure head and the water levels in each tank at the end of simulation period must be higher than or equal to the water levels of that tank at the start of the simulation period (Equations 4 and 5).

$$
\begin{gathered}
P_{\mathrm{Min}, i} \leq P_{i, t} \leq P_{\mathrm{Max}, i} \\
F T W L_{j(t=M)} \geq I T W L_{j(t=0)}
\end{gathered}
$$

where, $P_{M i n, i}$ and $P_{M a x, i}$ are the minimum and maximum acceptable pressures for junction i, $P_{i, t}$ is the pressure of junction $i$ at time $t, i$ is the junction index ( $\mathrm{i}=1,2,3, \ldots \ldots, n)$, and $t$ is the time index $(\mathrm{t}=1,2,3, \ldots \ldots, M) . F T W L_{j(t=M)}$ and ITWL $_{j(t t 0)}$ are the final and initial tank water levels of $j$ th tank. The sum of the number of EPANET errors/warning messages and total nodal pressure and tanks water levels violations are considered as a penalty in the optimization model.

\section{PST}

The PST is a model-based DST and is available for users (PST, 2019). It is a dynamic tool that can handle four different pump scheduling formulation types, including on and off, time control, time-length control, and simple control (tank water levels), to generate an optimum pump operation schedule for water systems. For a selected schedule type, the PST has the capability to integrate a MO evolutionary optimization package (GANetXL) with an external hydraulic solver (EPAENT) (Rossman, 1999) to obtain an optimum schedule. The PST has been built on a single framework as an Excel add-in, (Fig. 2A) that has a user-friendly interface which allows the user to easily formulate the optimization problem based on their desired schedule type. In addition, the PST interface enables the user to use the tool as standalone for simulation of a WDS model based on a hydraulic model's input file and in the interactive mode by changing pump status on the interface.

To achieve the optimum pump schedule with considerations outlined in Section 2, the PST framework is developed as an Excel add-in (Fig. 2A). The framework contains a 


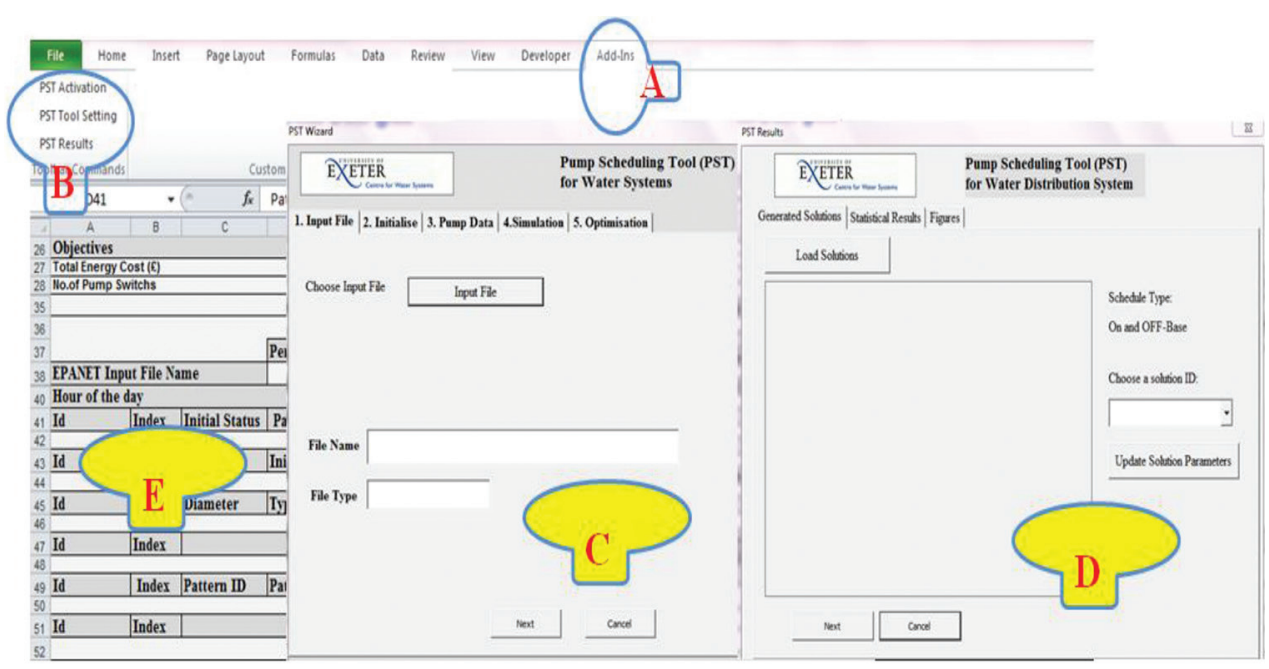

Fig. 2. The main components of the pump scheduling tool framework.

number of components, including a toolbar (Fig. 2B), a PST Configuration Wizard (Fig. 2C), PST result setting (Fig. 2D), and the interface spreadsheet of simulation model (Fig. 2E). The task of each component is described in the following sections.

\section{A. Toolbar}

The toolbar contains three control buttons including PST Activation, PST Tool Setting (Configuration Wizard), and PST results (Fig. 2B). The PST Configuration Wizard (Fig. 2C) is required to set all the parameters outlines above. After configuration of all the parameters and completion of an optimization run, the PST results (Fig. 2D) interface allows representation of the generated solutions and their corresponding parameters.

\section{B. PST Configuration Wizard}

The configuration wizard is an interactive interface which directs the user through the necessary steps and options to give user-defined simulation and optimization parameters required for any pump scheduling optimization problem executed within the PST. To configure a problem setting, the wizard has five main tabs: (1) Input file, (2) initialization, (3) Pump Data, (4) simulation, and (5) optimization.

Input file

By clicking on the "Input file" control button in the Input File tab (Fig. 2C), a search application window allows the user to easily browse for and choose an input file, and automatically imports it into the tool. An EPANET model in INP file format is required. Once the hydraulic model's (EPANET's) input file is plugged into the tool, the interface is automatically updated to simulate the model for further analysis. A warning message is issued by the PST when a non-EPANET input file type is chosen and hence the user is given an opportunity to choose another file.

\section{Initialization}

To perform the simulation and optimization model, the PST has two main worksheets ("Simulation Sheet" and
"Read Input File Sheet") as Excel spreadsheet models. The "Simulation Sheet" is the main simulation interface sheet and dedicated to the hydraulic simulation model outputs. The "Read Input File" sheet is designed to store most of the features of a WDS modeled by EPANET, which can be plugged into the tool as an EPANET input file. By initializing the model using the "Press to Initialize" control button (Fig. 3A), the system component parameters (e.g., number of pumps, tanks, valves, nodes, etc.) are retrieved from the external input file and populated in the "Read Input File Sheet." In addition, the tool has the ability to obtain other important parameters of the EPANET model and save them in the sheet, such as pattern (e.g., pump status and energy tariff pattern), time options (e.g., simulation duration time and simulation start time), and simple control rules.

A summary of statistical data plus an existing pump schedule type in the input file is presented in the initialization tab (Fig. 3A). For any new WDS model plugged to the tool, the worksheets are automatically updated: The previous data are removed, the hydraulic parameters of the new model components are retrieved and populated into the worksheets.

\section{Pump data}

In the Pump Data interface tab, three user-defined options (Fig. 3B) are available for system operators: Simulation (conducting only a hydraulic simulation run), modification and simulation (modifying the existing pump schedule using the interactive interface), and optimization (generating optimal schedule). The tool directs the user to perform the first two options in the interface sheet. A user-defined schedule type dropdown list in the Pump Data tab, shown in Fig. 3B-3, allows the user to choose a desired schedule from among four proposed alternative schedule types. A user-controlled "Add pump" button with corresponding dynamic dropdown list (Fig. 3B-3) allows the user to select desired pumps to be included in the optimization routine. The dropdown list is updated for any input file with related parameters such as pump ID, pump patterns, and control index. Once a pump ID is selected and the "Add pump" control button is clicked, the related parameters are added to 
the decision variable list in the simulation sheet. The number of decision variables is then automatically determined by the tool based on the number of pumps considered in the optimization process. Based on the selected pump schedule type, an appropriate range of each decision variable is set by PST and linked with the optimization part of the PST.

\section{Simulation}

The user can run the hydraulic model simulation for any EPANET input file in the simulation tab (Fig. 3B-1) using the "Run Simulation" control button. In addition, the interface sheet (Fig. 3B-2) allows the user to conduct trial and error simulations for user-defined manual schedule scenarios in the interactive interface.

\section{Optimization}

The optimization tab of the PST configuration wizard (Fig. 4) is dedicated for configuring optimization parameters. The PST has direct links with GANetXL that provides the optimization engine for the pump scheduling problem. The GA tab (Fig. 4A) allows the user to set GA parameters (e.g., optimization type, algorithm type, population size, etc.), and the configuration data are dynamically saved into the GANetXL configuration setting worksheet. The PST framework allows the user to pose a problem either as single or MO optimization. Cell range locations, ranges, and number of the decision variables can be automatically updated in the optimization parameters tab (Fig. 4B) for the selected schedule type and pumps in the optimization routine. The tool gives opportunity to select objective functions to be included in the optimization routine. The PST uses the setting defined in the optimization tabs (GA and optimization parameters). The constraints are also automatically evaluated during the optimization process. Since allowable nodal pressure ranges and considerations are case specific, the PST enables the user to decide inclusion of the pressure constraint (Fig. 4B). If this is considered, the user is required to set the minimum and maximum limits for nodal pressure. Based on the selected scheduling type, the tool sets the corresponding decision variables and evaluates the objective functions and constraints.

\section{PST Results Interface}

The PST results tab (shown in Fig. 5) is a visualization interface to display detailed information (e.g., decision

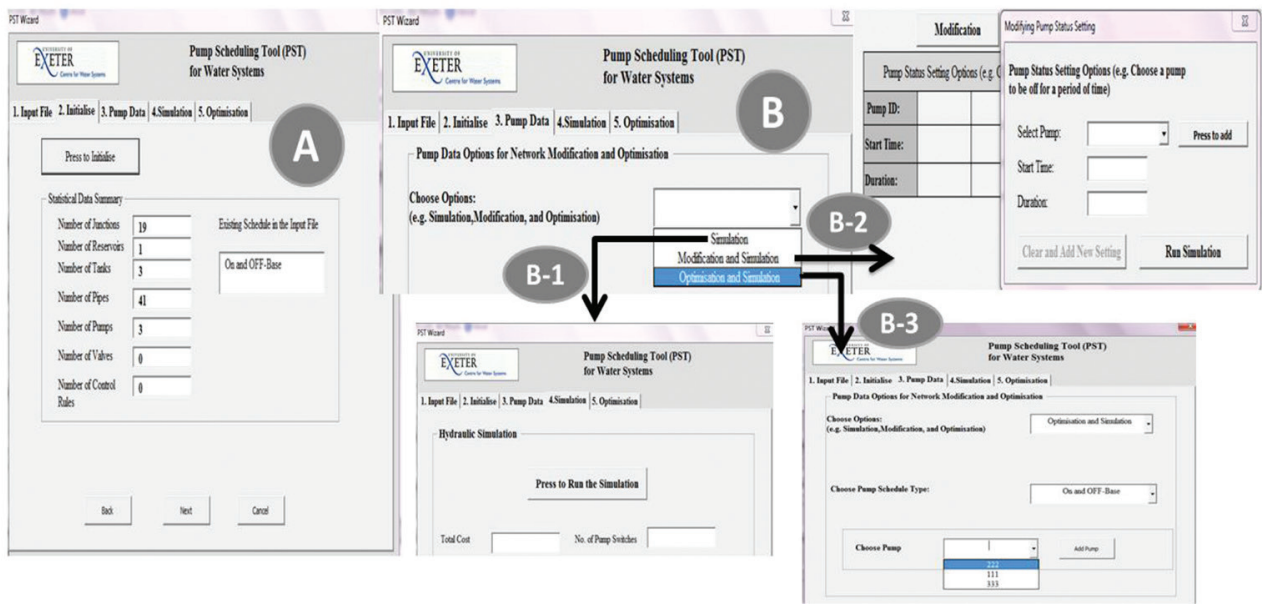

Fig. 3. The pump scheduling tool configuration wizard.

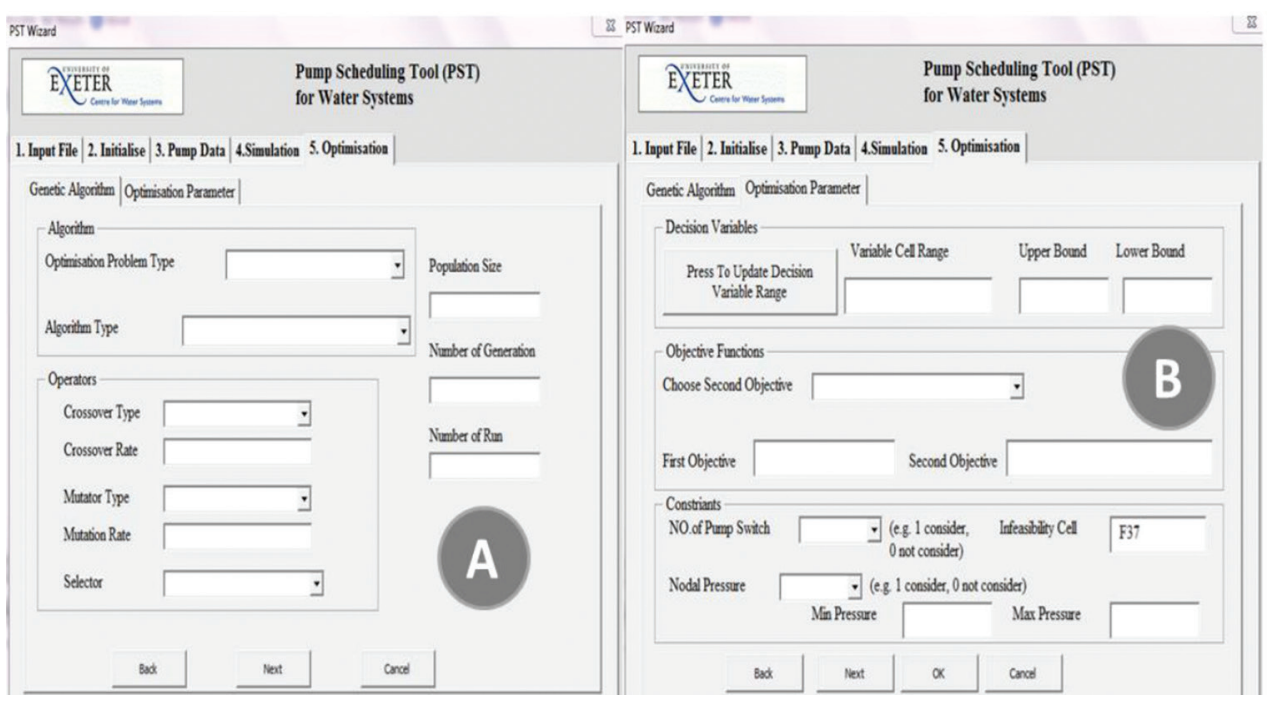

Fig. 4. The pump scheduling tool optimization tabs. 


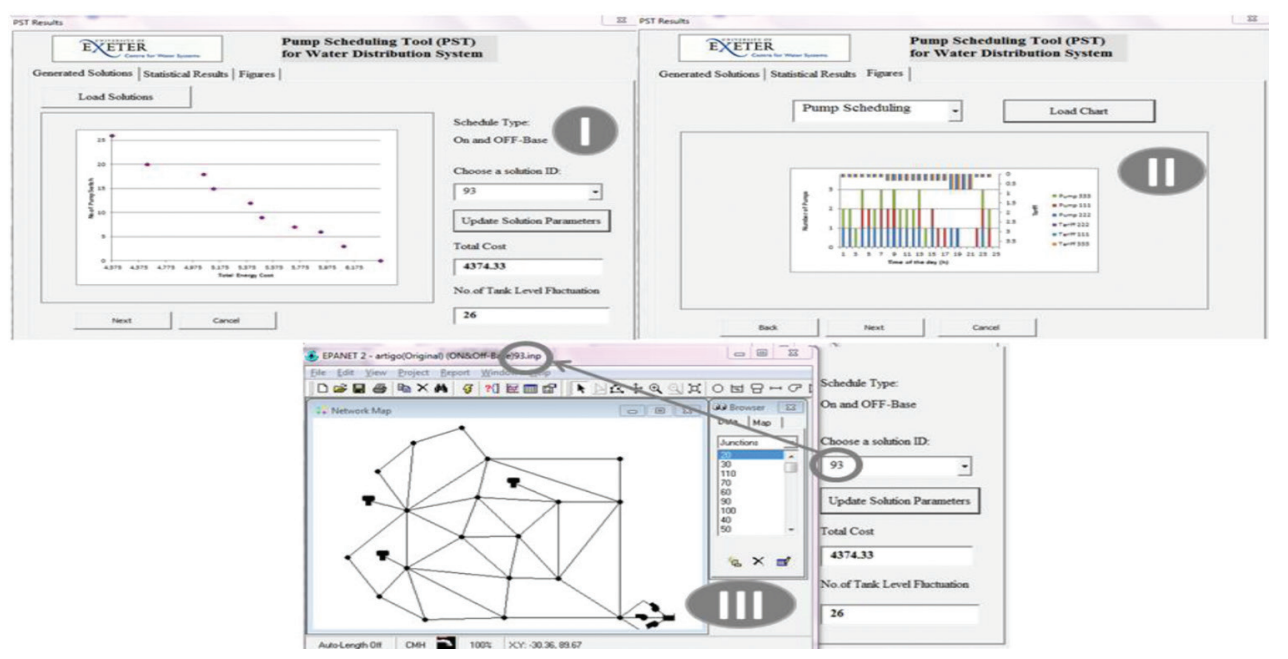

Fig. 5. The pump scheduling tool results interface.

variables, objective functions, and schedule) about the generated solutions. PST results can be invoked in the PST toolbar (Fig. 2B). The generated solutions in the result sheet can be easily displayed in a two-dimensional plot, just by clicking on the "load Solution" button.

The user can also interact with the generated solution parameters by choosing an individual solution ID (Fig. 5I) and clicking on the "Update Solution Parameters" button, which consequently loads the solution into the interface to demonstrate the change in decision variables, objective functions, and all the relevant information will be updated for that solution automatically. The solution will also be saved as an input file format in the current folder of the tool and automatically visualized in the EPANET interface (Fig. 5III).

The tool also facilitates the visual presentation of results (Fig. 5II) (e.g., water levels in the tanks over the operation period). When a solution is selected, the corresponding figures are automatically generated. The rich visualization and the analysis environment of the results representation by the PST helps the decision maker to analyze and assess the characteristics of solutions (e.g., through a Pareto-optimal front of solutions in the MO pump scheduling problem) and make informed decisions.

\section{Simulation Interface Spreadsheet Model}

The "Simulation Sheet" is a dynamic simulation interface sheet. This sheet is a second worksheet of the PST used to automatically make a link between the hydraulic model and the optimization model; and describe the fundamental parameters of the models. Presenting unknown intermediate parameters in the sheet would give the opportunity for users to explicitly monitor or investigate the model simulation results and optimization process. Each part of the sheet is dedicated to a specific analysis problem; designated cells for such analysis are dynamically sized for any EPANET input file. A general Dynamic Matrix (DM) is introduced in the sheet to manipulate those in-between variables for any given input file. The horizontal dimension (R) of the DM is equal to length of the EPS (e.g., 24 h), and the vertical dimension (C) is equal to the number of elements (e.g., number of pumps). The extent of the sheet size tends to be system specific, varying from system to system, depending on the number of components available in the system. For example, assuming an input file has three pumps and two tanks, then the size of the DM for pumps is 3 by 24 and for the tanks is 2 by 24 . Once the model is initialized, the process of directly loading statistical information of WDS elements from the input file sheet into the simulation sheet automatically updates the DMs as the format below.

$$
D M=\left[\left[\begin{array}{cccccc}
d m_{(1,1)} & d m_{(1,2)} & d m_{(1,3)} & \cdot & \cdot & d m_{(1, R)} \\
d m_{(2,1)} & d m_{(2,2)} & d m_{(2,3)} & \cdot & \cdot & d m_{(2, R)} \\
\cdot & \cdot & \cdot & \cdot & \cdot & \cdot \\
\cdot & \cdot & \cdot & \cdot & \cdot & \cdot \\
\cdot & \cdot & \cdot & \cdot & \cdot & \cdot \\
d m_{(C, 1)} & d m_{(C, 2)} & d m_{(C, 3)} & & & d m_{(1,1)}
\end{array}\right]_{(C * R)}\right.
$$

\section{Application of PST to Case Studies}

\section{A. Problem Description}

To demonstrate the capabilities of the PST, it has been applied to two most widely used WDS pump scheduling optimization problems. Anytown WDS is a well-known benchmark which was originally created by Walski, et al. (1987), and the modified version, so called Anytown (Modified) or AT(M) network, has been frequently used (Costa, de Athayde Prata and Ramos, 2015 and Rao and Alvarruiz, 2007) The second case is Richmond WDS which is a real one in the United Kingdom. The Richmond WDS was initially studied by van Zyl, Savić and Walters (2004) and later by Giacomello, Kapelan and Nicolini (2013). A full problem description can be found in van Zyl, Savić and Walters (2004). The EPANET input files for both networks are available from University of Exeter's Benchmark Library (CWS, 2018). EPANET network layout representations of 
both networks are shown in Fig. 6. Table I summarizes the general properties of both case study networks.

The main objective of both problems is to minimize the running costs of pumps while maintaining an acceptable level of service to customers. Both optimization problems are subject to some performance and physical limitations: (1) The water levels in the tanks should be between the minimum and maximum permitted levels throughout a simulation period of $24 \mathrm{~h},(2)$ at the end of the simulation period, the water levels in the tanks should be higher than the water levels at the start of the operation, and (3) minimum pressure requirement should be satisfied at all demand nodes. To ensure the search space is explored adequately, the optimization algorithm is

TABLE I

Properties of Case Study Networks

\begin{tabular}{lcc}
\hline \hline Network properties & Anytown & Richmond \\
\hline Number of reservoirs & 1 & 1 \\
Number of storage tanks & 3 & 6 \\
Number of pumps & 3 & 7 \\
Number of energy tariff patterns & 1 & 7 \\
Time horizon $(\mathrm{h})$ & 24 & 24 \\
Search space size & $2^{\left(3^{*} 24\right)} \approx 4.73 \times 10^{21}$ & $2^{\left(7^{*} 24\right)} \approx 3.74 \times 10^{50}$ \\
\hline \hline
\end{tabular}

run for 25,000 and 50,000 fitness function evaluations for Anytown and Richmond WDS, respectively.

\section{RESUlts AND Discussion}

The PST successfully managed to produce a Pareto front containing a set of feasible solutions for different pump schedule types for each network. Figs. 7 and 8 demonstrate the results produced by the PST, presenting four sets of Pareto fronts of non-dominated solutions generated based on four pump scheduling approaches for Anytown and Richmond WDS, respectively. The results correspond to only a single run of the optimization model, and all the solutions along the Pareto front curves are fully feasible, with no violation of any constraint during the simulation period. As can be observed in Fig. 8, utilization of various schedule strategies produces different sets of solutions. It can be also seen that the achieved solutions using time-length control and on and off schedules are better than the other solutions obtained using time control and simple control (tank water levels) approaches. This shows that various way of handlings and formulations of decision variables in a problem by an optimization algorithm can affect quality of the solution. The

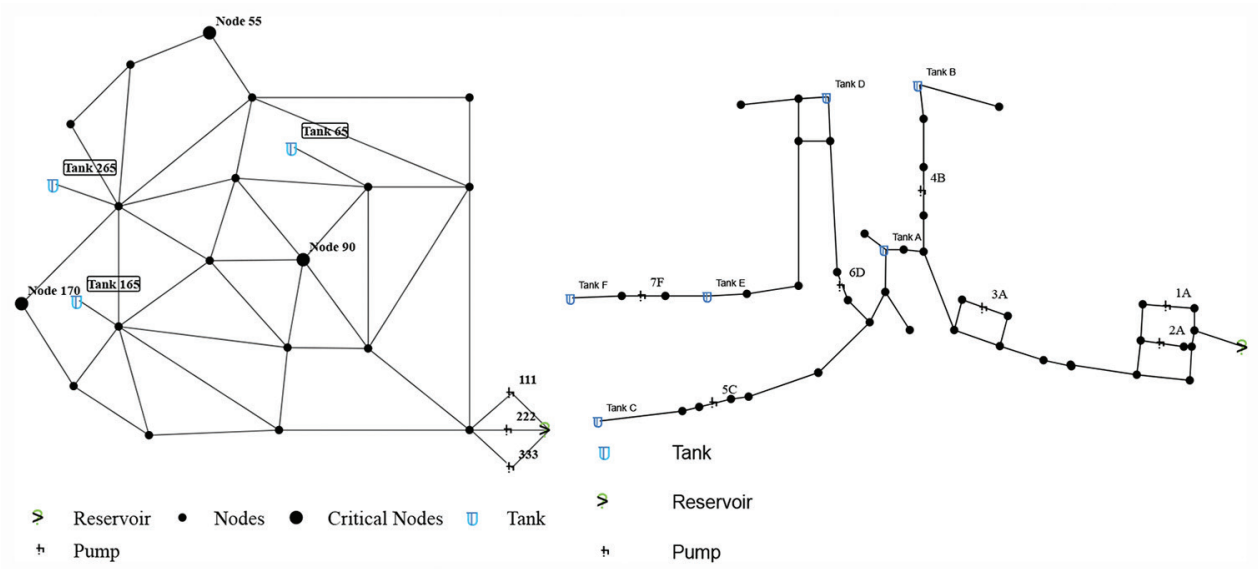

Fig. 6. Schematic representation of (1) Anytown and (2) Richmond networks.

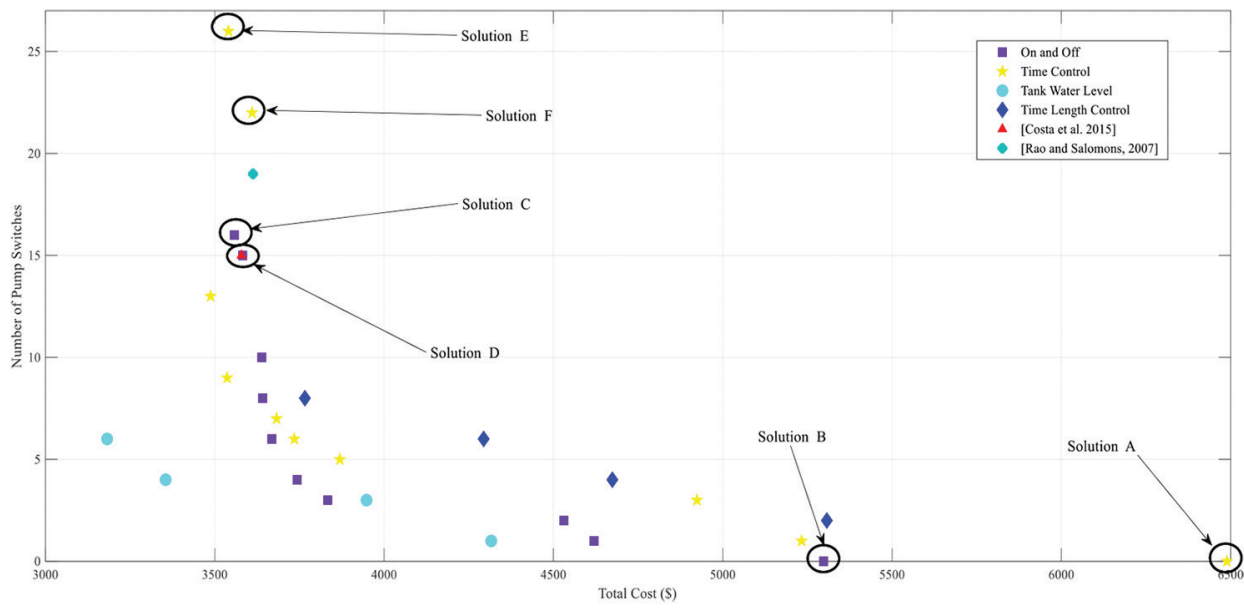

Fig. 7. Pareto fronts of the Anytown water distribution systems problem. 
result for the Anytown problem shows that the on and offbased approach has advantages over the other approaches. As can be shown in Fig. 7, Solutions A and B have the same (zero) number of pump switches, whereas Solution B on the on and off curve is much cheaper than Solution A on the time control curve. These demonstrate the usefulness of the PST in solving pump scheduling problems by considering different types of control strategies and helping the decisionmaker to make quantitative comparison between them.

To assess the robustness of the tool, the results are compared with those reported in the literature, as shown in Table II. The best presented solution for Anytown network by Rao and Salomons (2007) had a cost of $\$ 3612.84$ and 19 pump switches. This was later improved by Costa, de

TABLE II

List OF SOLUTIONS

\begin{tabular}{lcc}
\hline \hline Solution & Total cost (\$) & No. of pump switch \\
\hline C & 3557.70 & 16 \\
D & 3582.16 & 15 \\
Rao and Salomons & 3612.84 & 19 \\
Costa, et al. & 3578.66 & 15 \\
\hline \hline
\end{tabular}

Athayde Prata and Ramos (2015) with a reduced cost of $\$ 3578.66$ and fewer pump switches (i.e., 15). Solution $\mathrm{C}$ in Fig. 7 has a cost of $\$ 3557.70$ and 16 pump switches, which is $1.52 \%$ lower cost than the solution presented by Rao and Salomons (2007) and $0.59 \%$ less than the solution reported by Costa, de Athayde Prata and Ramos (2015). The number of pump switches for Solution D (Fig. 7) is 15 which is less than and equal to the number of pump switches presented by Rao and Salomons (2007) and Costa, de Athayde Prata and Ramos (2015), respectively. This solution has a cost of $\$ 3582.16$. Solution $\mathrm{E}$ on time control curve, Fig. 7 , is also $1.07 \%$, and $2 \%$ cheaper than the solutions reported by Rao and Salomons (2007) and Costa, de Athayde Prata and Ramos (2015), respectively.

Finally, Figs. 9 and 10 are automatically produced by the PST for Solutions C, D, E, and F in Fig. 7. These figures show the variation of water levels in the three tanks and the corresponding number of pumps operating during the course of the day in the Anytown network for each solution. Both figures show that water level in all tanks fluctuate between minimum and maximum acceptable levels throughout the

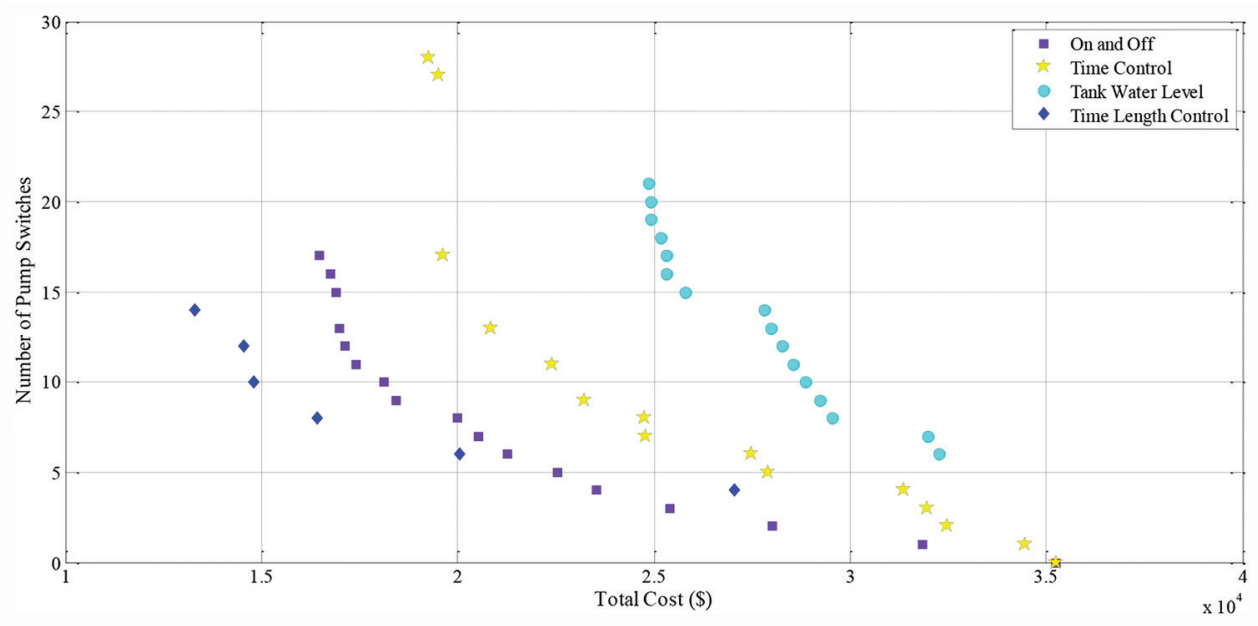

Fig. 8. Pareto fronts of the Richmond water distribution systems problem.
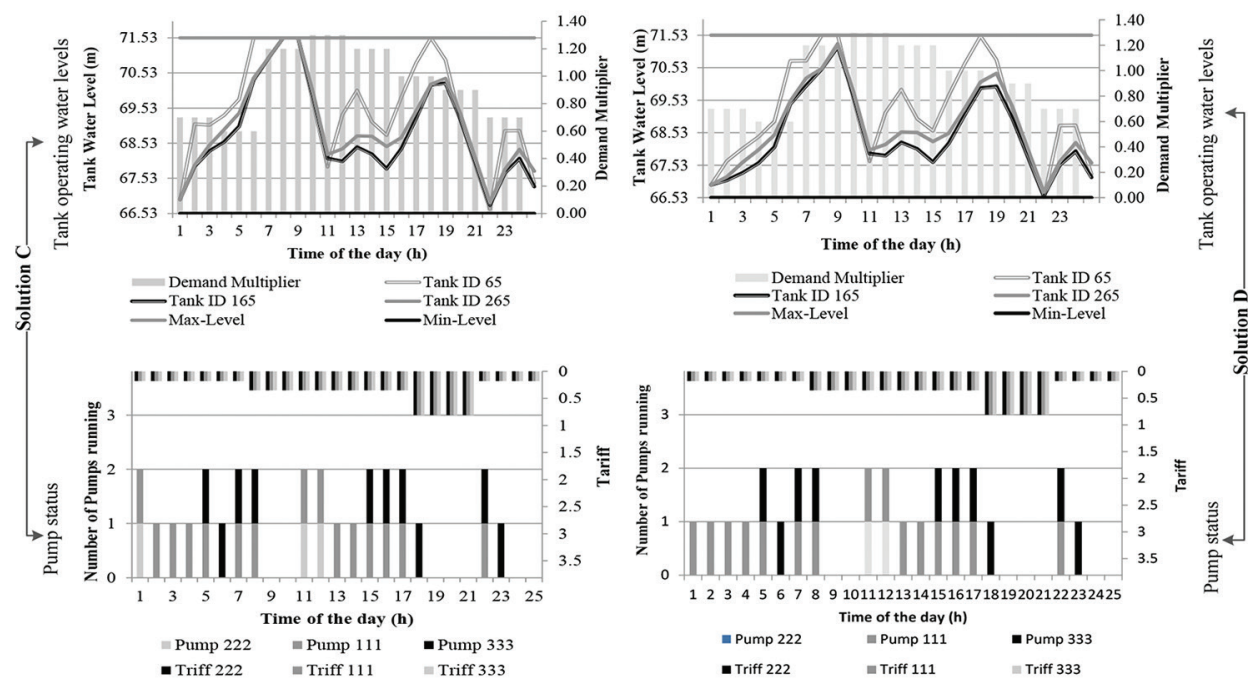

Fig. 9. Tank water levels and pump status for Solutions C and D for Anytown water distribution systems. 


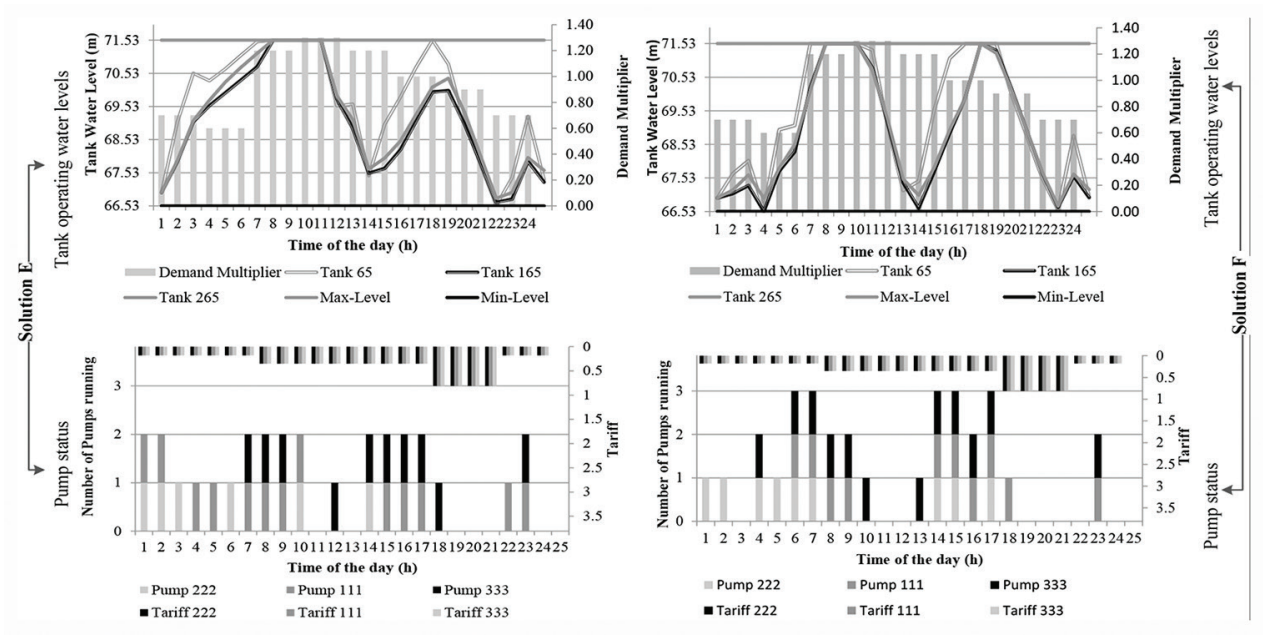

Fig. 10. Tank water levels and pump status for Solutions E and F for Anytown water distribution systems.

day, indicating short water residence times within the tanks which cause less water quality degradation. Turning off the pumps and supplying water from the tanks during peak water demand period (e.g., during early morning and late afternoon), as well as shifting heavy pumping load and filling up the tanks during cheaper energy periods (e.g., after mid-night) can also be observed in both Figs. 9 and 10. The results demonstrate the ability of the tool to effectively generate high-quality practical solutions for WDS pump scheduling problems using various schedule techniques in a single optimization run.

\section{CONCLUSION}

The operating cost of pumps in a WDS represents a substantial part of the total expenditure incurred in the operation of WDS. A dynamic DSS tool, the PST developed in a very sophisticated Excel spreadsheet-based model, is presented in this paper that supports four alternative pump scheduling schemes (on and off, time control, time-length control, and simple control [Tank water levels]) for WDS. It has the capability to generate and present pump scheduling solutions for a given problem. The tool includes linkage between an optimization algorithm with the EPS model to evaluate the impact of various pumps scheduling on the system performance over the operation period and calculate the objective function values. The easy-to-use interface of the tool offers simulation of WDS model based on: (1) A hydraulic model input file, (2) an interactive interface which can be modified by the user, and (3) pump operation generated by the optimization algorithm. The "result interface" of the PST also allows decision-makers to easily access detailed parameters of the generated solutions.

The PST application to two different water distribution networks shows a powerful decision support tool which requires no changes to the tool/interface as the tool automatically updates all the information based on the EPANET input file. Furthermore, the dynamic nature of the tool allows four different types of pump scheduling approaches in a single framework with no changes to the tool or interface. The generated results show that the tool is also able to deliver high-quality practical solutions for a given pump scheduling problem in comparison to the solutions reported in the literature. Future possible extension of this work could be considering variable speed pumps in the optimization of pump scheduling problems.

\section{ACKNOWLEDGMENTS}

This work forms part a collaborative research project between the UK and Chile, funded by the UK Government's prosperity fund. The PST description and its availability can be found in this link (http://emps.exeter.ac.uk/engineering/research/cws/ resources/pst/). The GANetXL can also found in here (http:// emps.exeter.ac.uk/engineering/research/cws/resources/ganetxl/).

\section{CONFLICTS OF INTEREST}

The authors declare no conflicts of interest.

\section{REFERENCES}

Abdallah, M., and Kapelan, Z., 2017. Iterative extended lexicographic goal programming method for fast and optimal pump scheduling in water distribution networks. Journal of Water Resources Planning and Management, 143(11), p.04017066.

Alighalehbabakhani, F., Miller, C., Abkenar, S., Fracasso, P., Jin, S., and McElmurry, S., 2015. Comparative evaluation of three distinct energy optimization tools applied to real water network (Monroe). Sustainable Computing: Informatics and Systems, 8, pp.29-35.

Amirabdollahian, M., and Mokhtari, M., 2015. Optimal Design of pumped water distribution networks with storage under uncertain hydraulic constraints. Water Resources Management, 29(8), pp.2637-2653.

Bagirov, A.M., Barton, A.F., Mala-Jetmarova, H., Al Nuaimat, A., Ahmed, S.T., Sultanova, N., and Yearwood, J., 2013. An algorithm for minimization of pumping costs in water distribution systems using a novel approach to pump scheduling. Mathematical and Computer Modelling, 57(3-4), pp.873-886.

Behandish, M., and Wu, Z., 2014. Concurrent pump scheduling and storage level optimization using meta-models and evolutionary algorithms. Procedia Engineering, 70, pp.103-112. 
Bene, J.G., and Hos, C.J., 2012. Finding least-cost pump schedules for reservoir filling with a variable speed pump. Journal of Water Resources Planning and Management, 138(6), pp.682-686.

Blinco, L., Simpson, A., Lambert, M., and Marchi, A., 2016. Comparison of pumping regimes for water distribution systems to minimize cost and greenhouse gases. Journal of Water Resources Planning and Management, 142, p.04016010.

Choi, Y.H., and Kim, J.H., 2019. Self-adaptive models for water distribution system design using single-/multi-objective optimization approaches. Water, 11(6), p.1293.

Costa, L., de Athayde Prata, B., Ramos, H., and de Castro, M., 2015. A branchand-bound algorithm for optimal pump scheduling in water distribution networks. Water Resources Management, 30(3), pp.1037-1052.

CWS, 2018. Richmond Network Benchmark at Centre for Water Systems. Available from: http://www.emps.exeter.ac.uk/engineering/research/cws/ resources/benchmarks/operation/richmond.php. [Last accessed on $2018 \mathrm{Jul} 07$ ].

De Corte, A., and Sörensen, K., 2013. Optimisation of gravity-fed water distribution network design: A critical review. European Journal of Operational Research, 228(1), pp.1-10.

Farmani, R., Ingeduld, P., Savic, D., Walters, G., Svitak, Z., and Berka, J., 2007. Real-time modelling of a major water supply system. Water Management, 160(2), pp.103-108.

Fayzul, M., Pasha, K., and Lansey, K., 2014. Strategies to develop warm solutions for real-time pump scheduling for water distribution systems. Water Resources Management, 28(12), pp.3975-3987.

Fracasso, P., Barnes, F., and Costa, A., 2013. Energy cost optimization in water distribution systems using markov decision processes. In: International Green Computing Conference Proceedings.

Ghaddar, B., Naoum-Sawaya, J., Kishimoto, A., Taheri, N., and Eck, B., 2015. A Lagrangian decomposition approach for the pump scheduling problem in water networks. European Journal of Operational Research, 241(2), pp.490-501.

Giacomello, C., Kapelan, Z., and Nicolini, M., 2013. Fast hybrid optimization method for effective pump scheduling. Journal of Water Resources Planning and Management, 139(2), pp.175-183.

Hashemi, S.S., Tabesh, M., and Ataeekia, B., 2014. Ant-colony optimization of pumping schedule to minimize the energy cost using variable-speed pumps in water distribution networks. Urban Water Journal, 11(5), pp.335-347.

Housh, M., and Salomons, E., 2018. Optimal dynamic pump triggers for cost saving and robust water distribution system operations. Journal of Water Resources Planning and Management, 145(2), p.04018095.

Jung, D., Kang, D., Kang, M., and Kim, B., 2014. Real-time pump scheduling for water transmission systems: Case study. KSCE Journal of Civil Engineering, 19(7), pp.1987-1993.

Kougias, I.P., and Theodossiou, N.P., 2013. Multi-objective pump scheduling using harmony search algorithm (HAS) and polyphonic HSA. Water Resources Management, 27(5), pp.1249-1261.

Kurian, V., Chinnusamy, S., Natarajan, A., Narasimhan, S., and Narasimhan, S., 2018. Optimal operation of water distribution networks with intermediate storage facilities. Computer Aided Chemical Engineering, 119, pp.215-227.

León-Celi, C., Iglesias-Rey, P., Martínez-Solano, F., and Mora-Melia, D., 2016. A methodology for the optimization of flow rate injection to looped water distribution networks through multiple pumping stations. Water, 8(12), p.575.

López-Ibáñez, M., 2009. Operational Optimisation of Water Distribution Networks, Ph.D. Thesis. Edinburgh Napier University, United Kingdom.

López-Ibáñez, M., Prasad, T., and Paechter, B., 2008. Ant colony optimization for optimal control of pumps in water distribution networks. Journal of Water Resources Planning and Management, 134(4), pp.337-346.

López-Ibáñez, M., Prasad, T.D., and Paechter, B., 2011. Representations and evolutionary operators for the scheduling of pump operations in water distribution networks. Evolutionary Computation, 19(3), pp.429-467.
Makaremi, Y., Haghighi, A., and Ghafouri, H., 2017. Optimization of pump scheduling program in water supply systems using a self-adaptive NSGA-II; a review of theory to real application. Water Resources Management, 31(4), pp.1283-1304.

Mala-Jetmarova, H., Barton, A., and Bagirov, A., 2015. Erratum for exploration of the trade-offs between water quality and pumping costs in optimal operation of regional multiquality water distribution systems. Journal of Water Resources Planning and Management, 141(2), p.08014001.

Mala-Jetmarova, H., Sultanova, N., and Savic, D., 2017. Lost in optimisation of water distribution systems? A literature review of system operation. Environmental Modelling and Software, 93, pp.209-254.

Mala-Jetmarova, H., Sultanova, N., and Savic, D., 2018. Lost in optimisation of water distribution systems? A literature review of system design. Water, 10(3), p.307.

Marchi, A., Simpson, A., and Lambert, M., 2016. Optimization of pump operation using rule-based controls in EPANET2: New ETTAR toolkit and correction of energy computation. Journal of Water Resources Planning and Management, 142(7), p.04016012.

Matott, L., Tolson, B., and Asadzadeh, M., 2012. A benchmarking framework for simulation-based Optimization of environmental models. Environmental Modelling and Software, 35, pp.19-30.

Menke, R., Abraham, E., Parpas, P., and Stoianov, I., 2016. Exploring optimal pump scheduling in water distribution networks with branch and bound methods. Water Resources Management, 30(14), pp.5333-5349.

Naoum-Sawaya, J., Ghaddar, B., Arandia, E., and Eck, B., 2015. Simulationoptimization approaches for water pump scheduling and pipe replacement problems. European Journal of Operational Research, 246(1), pp.293-306.

Nowak, D., Krieg, H., Bortz, M., Geil, C., Knapp, A., Roclawski, H., and Böhle, M., 2018. Decision support for the design and operation of variable speed pumps in water supply systems. Water, 10, p.734.

Odan, F., Ribeiro Reis, L., and Kapelan, Z., 2015. Real-time multiobjective optimization of operation of water supply systems. Journal of Water Resources Planning and Management, 141(9), p.04015011.

PST, 2019. PST, Centre for Water Systems, University of Exeter. Available from: http://www.emps.exeter.ac.uk/engineering/research/cws/resources/pst. [Last accessed on 2019 Mar 02].

Rao, Z., and Alvarruiz, F., 2007. Use of an artificial neural network to capture the domain knowledge of a conventional hydraulic simulation model. Journal of Hydroinformatics, 9(1), pp.15-24.

Rao, Z., and Salomons, E., 2007. Development of a real-time, near-optimal control system for water-distribution networks. Journal of Hydroinformatics, 9(1), pp.25-38.

Rossman, L., 1999. The EPANET programmer's toolkit for analysis of water distribution systems. In: Wilson, E.M., editors. $2^{\text {th }}$ Annual Water Resources Planning and Management Conference No. 1-10. American Society of Civil Engineering (ASCE), Tempe, Arizona, United States.

Sadatiyan, A.S., and Miller, C., 2017. PEPSO: Reducing electricity usage and associated pollution emissions of water pumps. Water, 9(9), p.640.

Sadatiyan, A.S., and Miller, C., 2019. PEPSO: Reducing electricity usage and associated pollution emissions of water pumps. Water, 9(9), p.640.

Savić, D.A., Bicik, J., and Morley, M.S., 2013. A DSS generator for multiobjective optimisation of spreadsheet based models. Environmental Modelling and Software, 26(5), pp.551-561.

Siew, C., Tanyimboh, T., and Seyoum, A., 2016. Penalty-free multi-objective evolutionary approach to optimisation of anytown water distribution network. Water Resources Management, 30(11), pp.3671-3688.

Skworcow, P., Paluszczyszyn, D., Ulanicki, B., Rudek, R., and Belrain, T., 2014. Optimisation of pump and valve schedules in complex large-scale water distribution systems using GAMS modelling language. Procedia Engineering, 70, pp.1566-1574. 
Stokes, C., Simpson, A., and Maier, H., 2015. A computational software tool for the minimization of costs and greenhouse gas emissions associated with water distribution systems. Environmental Modelling and Software, 69, pp.452-467.

Tsai, F., Katiyar, V., Toy, D., and Goff, R., 2008. Conjunctive management of large-scale pressurized water distribution and groundwater systems in semiarid area with parallel genetic algorithm. Water Resources Management, 23(8), pp.1497-1517.

Tsoukalas, I., Kossieris, P., Efstratiadis, A., and Makropoulos, C., 2016. Surrogate-enhanced evolutionary annealing simplex algorithm for effective and efficient optimization of water resources problems on a budget. Environmental Modelling and Software, 77, pp.122-142.

van Zyl, J.E., Savić, D.A., and Walters, G.A., 2004. Operational optimization of water distribution systems using a hybrid genetic algorithm. Journal of Water Resources Planning and Management, 130(2), pp.160-170.
Vieira, B., Mayerle, S., Campos, L., and Coelho, L., 2019. Optimizing drinking water distribution system operations. European Journal of Operational Research, 280(3), pp.1-34.

Walski, T., Chase, D., and Savic, D., 2003. Advance Water Distribution Modeling and Management. $1^{\text {st }}$ ed. Haestad Press, New York, USA.

Walski, T.M., Brill, E.D., Gessler, J., Goulter, I.C., Jeppson, R.M., Lansey, K., Lee, H.L., Liebman, J.C., Mays, L., Morgan, D.R., and Ormsbee, L., 1987. Battle of the network models: Epilogue. Journal of Water Resources Planning and Management, 113(2), pp.191-203.

Wang, J.Y., Chang, T.P., and Chen, J.S., 2019. An enhanced genetic algorithm for bi-objective pump scheduling in water supply. Expert Systems with Applications, 36, pp.10249-10258.

Zhang, L., and Zhuan, X., 2018. Optimisation on the VFDs' operation for pump units. Water Resources Management, 33(1), pp.355-368. 